\title{
NGC 3934: a shell galaxy in a compact galaxy environment
}

\author{
D. Bettoni ${ }^{1}$, G. Galletta ${ }^{2}$, R. Rampazzo ${ }^{1}$, A. Marino ${ }^{1}$, P. Mazzei $^{1}$, and L. M. Buson ${ }^{1}$
}

\author{
1 INAF, Osservatorio Astronomico di Padova, Vicolo dell'Osservatorio 5, 35122 Padova, Italy \\ e-mail: [daniela.bettoni; antonietta.marino;roberto.rampazzo;paola.mazzei;lucio.buson]@oapd.inaf.it \\ 2 Dipartimento di Astronomia, Universitá di Padova, Vicolo dell'Osservatorio 3, 35122 Padova, Italy \\ e-mail: giuseppe.galletta@unipd.it
}

Received 19 April 2011 / Accepted 5 August 2011

\begin{abstract}
Context. Mergers/accretions are considered the main drivers of the evolution of galaxies in groups. We investigate the NGC 3933 poor galaxy association that contains NGC 3934, which is classified as a polar-ring galaxy.

Aims. The multi-band photometric analysis of NGC 3934 allows us to investigate the nature of this galaxy and to re-define the NGC 3933 group members with the aim to characterize the group's dynamical properties and its evolutionary phase.

Methods. We imaged the group in the far (FUV, $\lambda_{\text {eff }}=1539 \AA$ ) and near (NUV, $\lambda_{\text {eff }}=2316 \AA$ ) ultraviolet (UV) bands of the Galaxy Evolution Explorer (GALEX). From the deep optical imaging we determined the fine structure of NGC 3934. We measured the recession velocity of PGC 213894 which shows that it belongs to the NGC 3933 group. We derived the spectral energy distribution (SED) from FUV to far-IR emission of the two brightest members of the group. We compared a grid of smooth particle hydrodynamical (SPH) chemo-photometric simulations with the SED and the integrated properties of NGC 3934 and NGC 3933 to devise their possible formation/evolutionary scenarios.

Results. The NGC 3933 group has six bright members: a core composed of five galaxies, which have Hickson's compact group characteristics, and a more distant member, PGC 37112. The group velocity dispersion is relatively low ( $\left.157 \pm 44 \mathrm{~km} \mathrm{~s}^{-1}\right)$. The projected mass, from the NUV photometry, is $\sim 7 \times 10^{12} M_{\odot}$ with a crossing time of 0.04 Hubble times, suggesting that at least in the center the group is virialized. We do not find evidence that NGC 3934 is a polar-ring galaxy, as suggested by the literature, but find that it is a disk galaxy with a prominent dust-lane structure and a wide type-II shell structure.

Conclusions. NGC 3934 is a quite rare example of a shell galaxy in a likely dense galaxy region. The comparison between physically motivated SPH simulations with multi-band integrated photometry suggests that NGC 3934 is the product of a major merger.
\end{abstract}

Key words. galaxies: elliptical and lenticular, cD - galaxies: interactions - galaxies: fundamental parameters - galaxies: formation galaxies: evolution

\section{Introduction}

Owing to the low galaxy velocity dispersion, accretions and major mergers are considered the main drivers of the evolution of galaxies that inhabit low-density environments, such as small groups. Ram-pressure stripping (Gunn \& Gott 1972; Bekki 2009 , and references therein) and galaxy "harassment" (see e.g. Moore et al. 1998) are the type of interactions that are thought to dominate within the core of dense clusters. The combination of the above type of interactions, certainly evolving with time, is at the origin of the segregation of galaxy morphology with radius, which has often been observed in clusters (Oemler 1974; Dressler 1980). In galaxy groups, also in more dense associations such as compact group of galaxies (CG), observations show a plethora of morphological properties (see e.g. Hickson 1982). Some CGs are composed only of spiral members; others, characterized by a low level of activity of their members, are dominated by E/S0 galaxies (ETGs hereafter) with old stellar populations (see e.g. Coziol et al. 2000). If interpreted in a hierarchical scenario as an evolutionary sequence, CGs would evolve from a more active (SF, AGN etc.) phase dominated by a population of late-type galaxies to a more relaxed and passive phase inhabited by ETGs.

In this picture, potentially "evolving" CG systems are interesting because they offer the possibility to investigate the multiplicity of mechanisms through which member galaxies and the group itself co-evolve. Zepf (1993) calculated that roughly 7\% of the galaxies in CGs are in the process of merging.

In this context, we here study the properties of the galaxies in the NGC 3933 group. NGC 3934, the second brighter galaxy in the group, has been classified as a polar-ring galaxy by Schweizer et al. (1983) and Whitmore et al. (1990). Polar-ring galaxies (PRGs) and related objects are systems frozen in a peculiar morphology, with matter rotating in two nearly perpendicular planes (see also Bertola et al. 1985; and Varnas et al. 1987). The Polar Ring Catalogue (PRC) lists more than 100 PRGs and candidate PRGs (Whitmore et al. 1990). Unfortunately, only a small fraction of them has been investigated. There is a general agreement that the formation of the polar rings is the result of some kind of "secondary event", such as an external accretion/merger. Numerical simulations have shown that polarring systems could be explained either by simple gas accretion (Reshetnikov et al. 1997; Bournaud \& Combes 2003) or by mergers of perpendicularly oriented disk galaxies (Bekki 1997, 1998). However, in the PRC most objects do not show this simple appearance with an edge-on central object and an edge-on polar ring. Instead they appear as systems going toward the stability of a fully mixed and symmetric configuration that crosses equilibrium states characterized by irregular structures. These latter systems, called in the PRC "polar-ring-related objects", provide a unique chance to investigate the PRG formation scenarios because the initial components have not yet disappeared. 

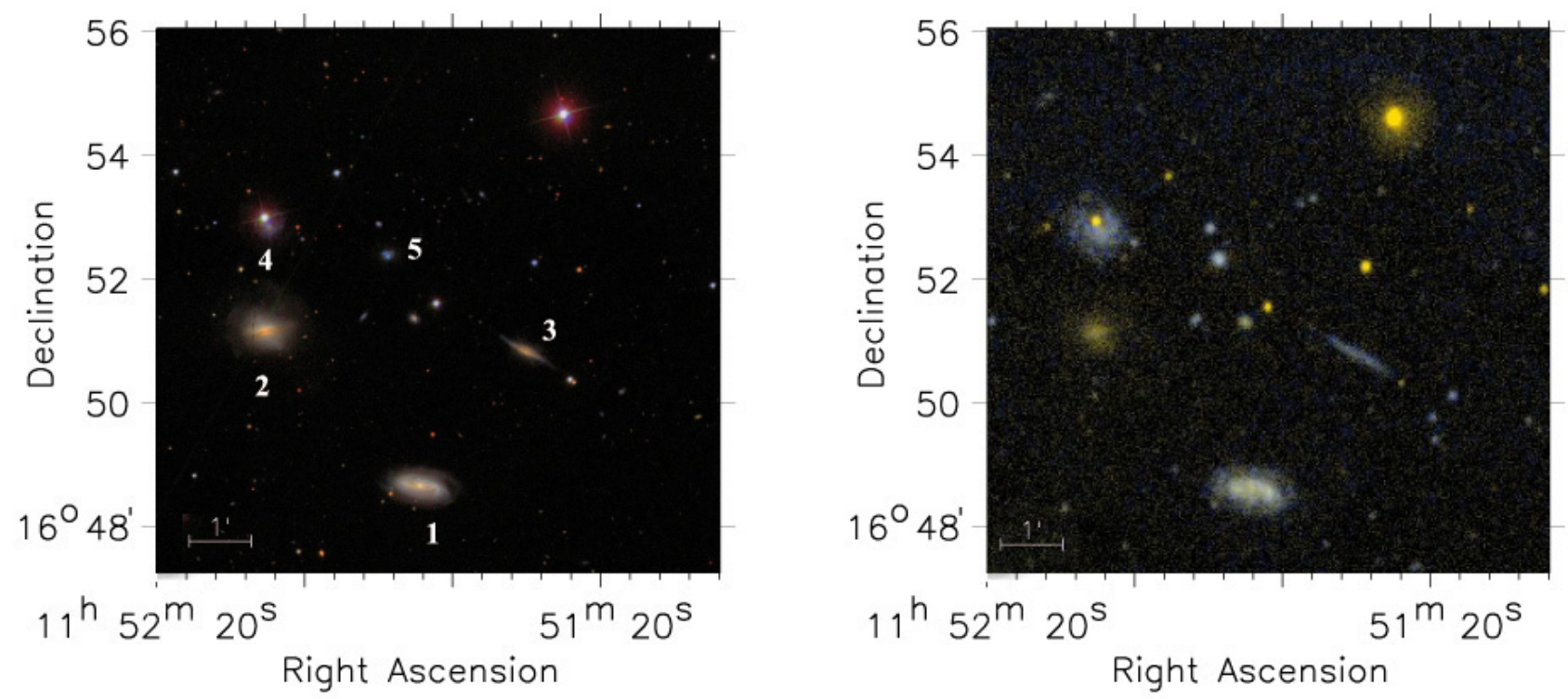

Fig. 1. The NGC 3933 group. (Left panel) Composite $u$ (blue), $g$ (yellow), $r$ (red) SDSS image. Right panel: full resolution, composite GALEX NUV (yellow) and FUV (blue) image. Galaxy members of the group are labeled as in Table 1. The galaxy labeled 6 is outside the field because it is $10.72^{\prime}$ southwest of NGC 3933 .

Table 1. Known members of the NGC 3933 group.

\begin{tabular}{llccccc}
\hline \hline$\#$ & Name & $\begin{array}{c}\text { RA } \\
{[\mathrm{deg}]}\end{array}$ & $\begin{array}{c}\text { Dec } \\
{[\mathrm{deg}]}\end{array}$ & $\begin{array}{c}\text { Morpho. } \\
\text { type }\end{array}$ & $\begin{array}{c}V_{\mathrm{h}} \\
{\left[\mathrm{km} \mathrm{s}^{-1}\right]}\end{array}$ & $\begin{array}{c}B_{\mathrm{T}} \\
{[\text { Vega }]}\end{array}$ \\
\hline 1 & NGC 3933 & 178.00845 & 16.80970 & $\mathrm{~S} 0-\mathrm{a}$ & $3734 \pm 10$ & $14.27 \pm 0.08$ \\
2 & NGC 3934 & 178.05255 & 16.85163 & Sab & $3779 \pm 4$ & $14.93 \pm 0.27$ \\
3 & UGC 6835 & 177.97860 & 16.84582 & - & $3549 \pm 17$ & $16.68 \pm 0.37$ \\
4 & PGC 213894 & 178.04985 & 16.87857 & & & $17.78 \pm 0.50$ \\
5 & SDSSJ115204.21+165217.3 & 178.01745 & 16.87164 & - & $3678 \pm 38$ & $17.68 \pm 0.50$ \\
6 & PGC 37112 & 177.90015 & 16.66425 & - & $4045 \pm 28$ & $16.67 \pm 0.37$ \\
\hline
\end{tabular}

Notes. Morphological type, the systemic heliocentric recession velocity and the $B$-band total magnitudes in the Vega-systems are derived from the HYPERLEDA data-base.

If they are the results of mergers, it may still be possible to recognize the progenitors in the unrelaxed remnants, and one may attempt to reconstruct their formation events. The NGC 3934 morphology falls in the category of polar-ring-related objects.

On the other hand NGC 3934 also possesses a wide shell system. It is also widely believed that shell systems are produced by merger episodes (Dupraz \& Combes 1986; Weil \& Hernquist 1993). NGC 3934 is a gas-rich system: it was detected both in HI (van Driel et al. 2002) and CO (Galletta et al. 1997) and it is a bright IRAS source. It may then represent a case of "wet" merging within compact galaxy associations. This paper presents the properties of the NGC 3933 system, providing also evidence that its "core" is a compact group of galaxies.

The paper is organized as follows: photometric and spectroscopic observations, data reduction and techniques are described in Sect. 2. The results are presented in Sect. 3. In Sect. 4 we discuss the kinematical and dynamical analysis of the group and interpret the SED and the global properties of the brighter members in the sample, namely NGC 3933 and NGC 3934, using physically motivated SPH simulations. Our conclusions are presented in Sect. 5.

\section{Observations and data reduction}

In Table 1 we collect the data of the known members of NGC 3933 studied here. In the left panel of Fig. 1 we show the composite SDSS image of the "core" of the NGC 3933 group (galaxies are labeled according to Table 1), while in the right panel we show the composite GALEX image of the same field. PGC 213894, which is faint in the SDSS image is very bright in the UV one. Contrary to this the presence of dust makes NGC 3934 very faint in the UV image.

Using the available recession velocities, the NGC 3933 galaxy group has four nearby companions, in projection. Two of them, NGC 3934 and UGC 6835, have a similar magnitude and size as NGC 3933. SDSSJ115204.21+165217.3 and PGC 037112 are noticeably fainter, and the latter is $\sim 10.72^{\prime}$ away from NGC 3933. At a distance of $\sim 40^{\prime}$ southwest of the NGC 3933 group "core" there is another spiral galaxy, UGC 6794, with a heliocentric velocity $\left(3457 \pm 7 \mathrm{~km} \mathrm{~s}^{-1}\right)$. HYPERLEDA reports 15 additional galaxies within $2 \mathrm{Mpc}$ from the group core and in the recession velocity range $3200 \leq V_{\text {hel }} \leq$ $4200 \mathrm{~km} \mathrm{~s}^{-1}$. These are distributed along a band oriented east to west; this structure will be discussed in Sect. 4.1

Optical spectroscopic observations have been performed with the twofold objective to determine the group membership by measuring the recession velocity of the spiral galaxy PGC 213894, 1.6' north of NGC 3934 (with a bright star superimposed) and to characterize the velocity dispersion of the polar-ring galaxy NGC 3934.

Far UV GALEX observations and deep optical imaging were performed to extend the galaxy spectral energy distribution to 
Table 2. UV total magnitudes of the group members.

\begin{tabular}{lcccccc}
\hline \hline Ident. & Type & $\begin{array}{c}\text { Major-axis } \\
{[\operatorname{arcmin}]}\end{array}$ & $\begin{array}{c}\text { Minor-axis } \\
{[\operatorname{arcmin}]}\end{array}$ & $\begin{array}{c}\text { PA } \\
{[\mathrm{deg}]}\end{array}$ & $\begin{array}{c}\text { FUV } \\
{[\mathrm{AB} \text { mag }]}\end{array}$ & $\begin{array}{c}\text { NUV } \\
{[\mathrm{AB} \mathrm{mag}]}\end{array}$ \\
\hline NGC 3933 & $5 \pm 1$ & 0.70 & 0.35 & 84.0 & $17.02 \pm 0.07$ & $16.50 \pm 0.04$ \\
NGC 3934 & $-2 \pm 1$ & 0.50 & 0.33 & 70.4 & $20.40 \pm 0.19$ & $18.71 \pm 0.06$ \\
UGC 6835 & $3 \pm 1$ & 0.50 & 0.12 & 64.9 & $19.83 \pm 0.12$ & $19.54 \pm 0.08$ \\
PGC 213894 & $4 \pm 1$ & 0.70 & 0.70 & 0.0 & $17.96 \pm 0.08$ & $17.59 \pm 0.04$ \\
SDSSJ115204.21+165217.3 & $10 \pm 1$ & 0.30 & 0.30 & 0.0 & $19.01 \pm 0.09$ & $18.67 \pm 0.06$ \\
PGC 037112 & $8 \pm 1.3$ & 0.32 & 0.28 & 2.0 & $18.00 \pm 0.07$ & $17.61 \pm 0.04$ \\
\hline
\end{tabular}

short wavelengths and to detail the fine structure of the polar ring of NGC 3934.

\subsection{Spectroscopic observations}

We measured the central velocity dispersion of NGC 3934 from a galaxy spectrum taken with ALFOSC@NOT (La Palma, Spain) in March 2001. We used ALFOSC which is equipped with a Ford-Loral $2024 \times 2024$ CCD with $15 \times 15 \mu$ m pixels and the grism \#13 with a wavelength range $\lambda \lambda=4800-5800 \AA$. We adopted a slit width of $2.5^{\prime \prime}$ that yields a resolution of $3.3 \AA$. The spectrum was obtained along PA $=26^{\circ}$ with an exposure time of $1800 \mathrm{~s}$. Two template stars for measuring the radial velocity and velocity dispersion, which are of spectral type G8 III (HD 65934) and K0 III (HD 132737), were also observed with the same spectral set-up.

In order to measure the redshift of the spiral galaxy PGC 213894, we obtained a spectrum at the Asiago Cima Ekar telescope in February 2007. We used the Asiago Faint Object Spectrograph and Camera (AFOSC) with grism \#4 with a wavelength range $\lambda \lambda=3500-7500 \AA$ and a $2.1^{\prime \prime}$ wide slit. This yields a dispersion of $4.9 \AA$ pixel $^{-1}$. The detector is a CCD Tektronix TK $1024 \times 1024$, with pixel size $24 \mu \mathrm{m}$, corresponding to a spatial scale along the slit of 0.473 arcsec pixel $^{-1}$. The spectrum was obtained along PA $=45^{\circ}$ with an exposure time of $1800 \mathrm{~s}$ and also crosses the bright star superimposed on the galaxy. The data reduction procedure, performed using the IRAF ${ }^{1}$ LONGSLIT package, was similar for both spectra. Scientific frames were corrected for bias and flat-field and calibrated using an $\mathrm{He} / \mathrm{Ar}$ arc-lamp.

No emission lines are present in the spectrum of NGC 3934 which was very noisy, because of dust in the central regions. For this reason, we co-added the spectrum in an aperture of $10^{\prime \prime}$. This may lead to an overestimate of the measured velocity dispersion if a rotation gradient is present. With our adopted distance, the coadded region corresponds to a physical region of $2.5 \mathrm{kpc}$; for an early-type galaxy this can lead to an overestimate of about $\sim 10 \%$, well within the errors of our measure. The velocity dispersion and the radial velocity were derived, using the Fourier quotient technique (Sargent et al. 1977; Bertola et al. 1984). We obtained the following results: $V_{r}=3740 \pm 88 \mathrm{~km} \mathrm{~s}^{-1}$ and $\sigma=169.5 \pm 19.5 \mathrm{~km} \mathrm{~s}^{-1}$. The galaxy recession velocity, corrected for heliocentric motion, agrees with values already available in the literature (see Table 1).

The spectrum of the spiral PGC 213894 shows the $\mathrm{O}[\mathrm{III}] \lambda 5007, \mathrm{H}_{\alpha}$ and [NII] $\lambda \lambda 6548-6583$ emission lines. All emission lines were fitted with a Gaussian profile and the final

\footnotetext{
1 IRAF is the Image Analysis and Reduction Facility made available to the astronomical community by the National Optical Astronomy Observatories, which are operated by the Association of Universities for Research in Astronomy (AURA), Inc., under contract with the US National Science Foundation.
}

measured galaxy radial velocity is the mean value with the error being the standard deviation. The velocity, corrected to the Sun, is $V_{r}=3542 \pm 40 \mathrm{~km} \mathrm{~s}^{-1}$, which is compatible with the average group velocity (see Table 5) and implies that the galaxy is a member of the group.

\subsection{UV and optical imaging}

The UV imaging was obtained with GALEX observatory (see Martin et al. 2005; Morrisey et al. 2005) in its ultraviolet bands FUV (1344-1786 ^) and NUV (1771-2831 ̊). The instrument consists of a $50 \mathrm{~cm}$ diameter modified Richey-Chretien telescope that has a very wide field of view (1.25 degrees diameter) and a spatial resolution of $\approx 4$ ".2 and 5.'3 FWHM in FUV and NUV respectively, sampled with $1^{\prime \prime} .5 \times 1^{\prime \prime} .5$ pixels.

The FUV and NUV images were obtained on March 27, 2006, by means of dedicated observations awarded to our team (GI1 program 59, P.I. D. Bettoni). The exposure times were 1683 sec in both bands (limiting magnitude in FUV/NUV 22.6/22.7 AB mag (Bianchi 2009)). A UV composite image of the group (FUV blu; NUV yellow) is shown in the right panel of Fig. 1.

We used FUV and NUV GALEX background-subtracted intensity images to compute the integrated photometry of the galaxies within elliptical apertures (see Table 2). Background counts were estimated from the sky-background image and the high-resolution relative response map provided by the GALEX pipeline. In Table 2 we report the morphological type following de Vaucouleurs et al. (1991), the major and minor axis length, the position angle of the major axis, and the total FUV and NUV magnitudes of the group members. They were computed as $m(\mathrm{AB})_{\mathrm{UV}}=-2.5 \times \log \mathrm{CR}_{\mathrm{UV}}+\mathrm{ZP}$, where $\mathrm{CR}$ is the dead-time corrected, flatfielded count rate, and the zero points are $\mathrm{ZP}=$ 18.82 and $\mathrm{ZP}=20.08$ in FUV and NUV, respectively (Morrissey et al. 2007).

Optical photometric observations were carried out in May 2000 with OIG@Telescopio Nazionale Galileo. The OIG was equipped with a mosaic of two thinned and back-illuminated EEV 42-80 CCDs, each with $2048 \times 4096$ pixels. The pixel size and pixel scale are $13.5 \mu \mathrm{m}$ and $0.072^{\prime \prime} /$ pixel respectively, corresponding to a total field of view of about $4.9^{\prime} \times 4.9^{\prime}$. The OIG data were resampled with a binning factor of two, corresponding to a spatial scale of $0.144^{\prime \prime} /$ pixel. Exposure times were $1200 \mathrm{~s}$ in the $R$ - and $B$-Johnson band filters. Photometric calibration was achieved using the observations of standard stars from the Landolt (1983) and Smith et al. (1991) lists. The seeing during the observations was $\sim 1.2$ arcsec. Figure 2 shows the color image of the OIG frame.

The standard CCD image reduction was performed using the IRAF package. This includes dark and bias subtraction, flat-field correction (we used a mean of several dome flats taken in the appropriate filter), and sky subtraction. The cosmetic defects and cosmic rays were excluded by median filtering and the projected 

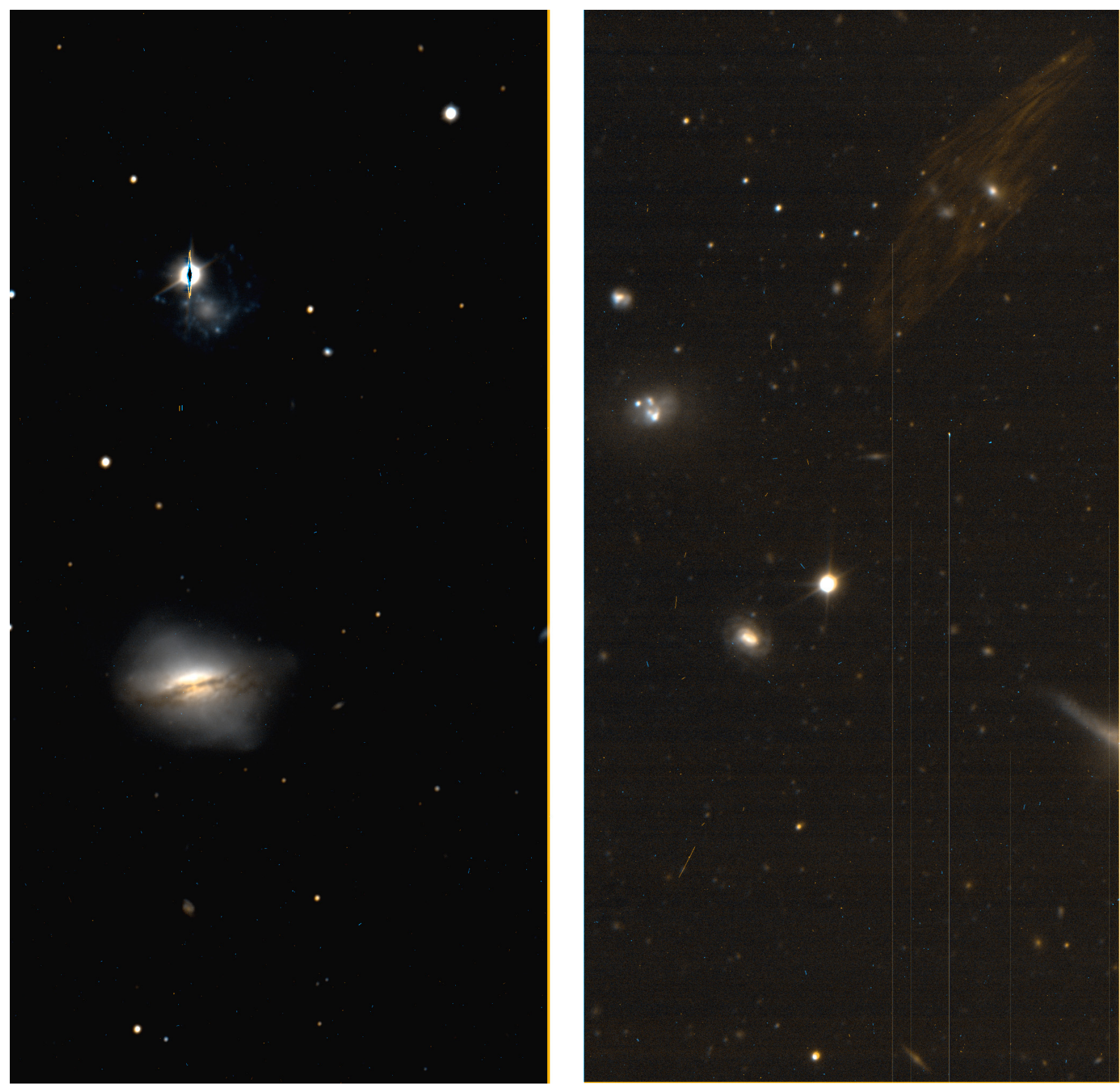

Fig. 2. Color composite image of NGC 3934 assembled from TNG+OIG images in the $B$ and $R$ bands, North is up while the East on the left of the image. In the top right of the figure is visible a defect generated by the CCD detector. The field of view is $4.9^{\prime} \times 4.9^{\prime}$.

stars by masking them with rectangular regions. The typical uncertainty of the background level is about $0.5 \%$.

The surface photometry was derived by means of the ELLIPSE package (Jedrzejewski 1987), which computes a Fourier expansion for each successive isophote, producing radial luminosity and geometrical profiles sampled in nested ellipses. These profiles are plotted versus galacto-centric distance along the circularized semiaxis in Fig. 5.

We obtained the detailed surface photometry of both NGC 3934 and of the new member of this group, PGC 213894. In order to extract the effective radius $r_{\mathrm{e}}$ and the effective surface brightness $\mu_{\mathrm{e}}$ of these galaxies, we performed a best fit of their surface brightness profiles. The images, the luminosity, ellipticity, and position angle profiles suggest that these galaxies have a disk component in addition to the bulge. We then use a model composed of a bulge, following the $r^{1} / 4$ law (de Vaucouleurs 1948), and an exponential disk (Freeman 1970).

PGC 213894 has a bright star (SDSS J115212.60+165255.4) at $11^{\prime \prime} \mathrm{NE}$ of the nucleus. This star, showing the typical spectral features of an M star, makes it difficult to fit ellipses to the galaxy image. In addition, the disk appears faint and the spiral arms dominate the $B$ - and $R$-images. To overcome these problems, we fitted not only the circularized luminosity profiles, but also the aperture photometry produced by ELLIPSE. We made on our theoretical profiles fit both the luminosity profile, $\mu(r)$, and the growth curve, $m(r)$. The central absorption is then minimized because it contributes less to the total value of integrated magnitude. Results of the best fitting, reported in Table 3, agree 
Table 3. Photometric data for NGC 3934, PGC 213894, and SDSSJ115204.21+165217.3. We assumed a group distance of 49.62 Mpc to compute the galaxy absolute magnitudes. Magnitudes are not corrected for galactic extinction.

\begin{tabular}{|c|c|c|c|c|c|c|c|}
\hline \multirow[b]{2}{*}{$\begin{array}{l}\text { Galaxy } \\
\text { name }\end{array}$} & \multirow[b]{2}{*}{ Band } & \multicolumn{2}{|c|}{ Bulge } & \multicolumn{2}{|c|}{ Disk } & \multirow[b]{2}{*}{$m_{\text {tot }}$} & \multirow[b]{2}{*}{$M_{\mathrm{tot}}$} \\
\hline & & $\begin{array}{c}r_{\mathrm{e}} \\
{[\operatorname{arcsec}]}\end{array}$ & $\begin{array}{c}\mu_{\mathrm{e}} \\
{\left[\mathrm{mag} / \mathrm{arcsec}^{2}\right]}\end{array}$ & $\begin{array}{c}r_{\mathrm{e}} \\
{[\operatorname{arcsec}]}\end{array}$ & $\begin{array}{c}\mu_{\mathrm{e}} \\
{\left[\mathrm{mag} / \mathrm{arcsec}^{2}\right]}\end{array}$ & & \\
\hline \multirow[t]{2}{*}{ NGC 3934} & $B$ & $9.0 \pm 5.5$ & $23.80 \pm 0.88$ & $15.5 \pm 0.2$ & $23.50 \pm 0.04$ & $14.43 \pm 0.29$ & -19.29 \\
\hline & $R$ & $8.0 \pm 4.6$ & $21.60 \pm 0.90$ & $16.0 \pm 0.5$ & $22.60 \pm 0.10$ & $13.03 \pm 0.28$ & -20.69 \\
\hline \multirow{2}{*}{ PGC 213894} & $B$ & $22.0 \pm 15.0$ & $26.40 \pm 0.78$ & $12.0 \pm 2.1$ & $25.00 \pm 0.25$ & $15.81 \pm 1.03$ & -17.91 \\
\hline & $R$ & $22.0 \pm 15.2$ & $25.80 \pm 0.80$ & $12.0 \pm 0.4$ & $23.80 \pm 0.07$ & $14.95 \pm 0.34$ & -18.77 \\
\hline \multirow[t]{2}{*}{ SDSSJ115204.21+165217.3 } & $B$ & - & - & - & - & $17.30 \pm 0.05$ & -16.20 \\
\hline & $R$ & - & - & - & - & $16.59 \pm 0.04$ & -16.90 \\
\hline
\end{tabular}

Table 4. Magnitudes and colors of the faint galaxies in the field.

\begin{tabular}{lccc}
\hline \hline Ident. & $m_{R}$ & $m_{B}$ & $B-R$ \\
\hline 1 & $19.32 \pm 0.073$ & $21.17 \pm 0.07$ & $1.78 \pm 0.101$ \\
2 & $21.41 \pm 0.212$ & $22.62 \pm 0.08$ & $1.21 \pm 0.226$ \\
3 & $21.27 \pm 0.042$ & $22.95 \pm 0.14$ & $1.68 \pm 0.146$ \\
4 & $20.24 \pm 0.103$ & $22.02 \pm 0.12$ & $1.78 \pm 0.158$ \\
5 & $20.91 \pm 0.030$ & $22.19 \pm 0.09$ & $1.28 \pm 0.094$ \\
6 & $21.45 \pm 0.100$ & $23.16 \pm 0.13$ & $1.71 \pm 0.164$ \\
7 & $21.60 \pm 0.049$ & $23.31 \pm 0.08$ & $1.71 \pm 0.094$ \\
8 & $20.02 \pm 0.055$ & $22.28 \pm 0.20$ & $2.26 \pm 0.207$ \\
9 & $20.40 \pm 0.076$ & $22.66 \pm 0.13$ & $2.26 \pm 0.150$ \\
\hline
\end{tabular}

with those available in HYPERLEDA ${ }^{2}$. In the right part of the OIG frame, 1.1' SW of SDSSJ115204.21+165217.3, the galaxy SDSSJ115202.45+165117.4 is also visible, a background object with a redshift $z=0.115$. Moreover many faint galaxies are visible in our image that may be related to the group. We measured the total $B$ - and $R$-magnitudes of these faint object and report them in Table 4. Despite their late-type morphology their $(B-R)$ color appears to be much redder than that of their morphological class (see e.g. the color profile of PGC 213894 in Fig. 6). We suggest that they are background galaxies.

\section{Results}

\subsection{Morphology and structure of member galaxies from optical OIG observations}

NGC 3934 has strong dust-lanes that irregularly obscur the inner and brighter regions of the galaxy. They are shown in Fig. 3 (right panel). Spurs and shells are visible in the inner parts, but especially in the outskirts of the galaxy. The $B-R$ color profiles for NGC 3934 and PGC 213894 are shown in Fig. 6. In NGC 3934, the galaxy outskirts have an average $B-R \sim 1.2$, that is not as blue as typical spiral arms although they are bluer than the central parts which is dominated by the dust-lane. As an example in the nearby spiral companion PGC 213894, where the average color of the arm region is $\sim 0.9$.

This irregularity is also visible from the data shown in the top panel of Fig. 5 where there is a rapid increase of the position angle $\left(\triangle \mathrm{PA} \sim 40^{\circ}\right)$ in the inner $15^{\prime \prime}$, followed by a monotonic decrease in the outskirts. Summarizing, we do not find signatures of either a polar-ring structure or of an inclined ring in the galaxy; the system is instead embedded in a complicated, type-II systems of inner and outer shells (see Prieur 1990). This shell system is reminiscent of that observed in the interacting S0 galaxy NGC 474 (see Fig. 1 in Rampazzo et al. 2006). However,

\footnotetext{
${ }^{2}$ http://leda.univ.lyon $1 . \mathrm{fr} /$
}

the strong dust-lane in addition to the system of shells points out that NGC 3934 is the product of a "wet" merging episode.

The $B$ - and $R$-band luminosity profiles of NGC 3934 show a disk. In the $B$-band we measure a typical disk central brightness $\mu_{0}=21.68$, similar to the classic value of $21.65 \pm 0.30$ found for a disk (Freeman 1970) with a scale length of 9.2" (see data in Table 3). Our total $B$-magnitude $m_{B}=14.43 \pm 0.29$ agrees with the values $m_{B}=14.27 \pm 0.08$ listed in the HYPERLEDA catalogue. The total absolute magnitude indicates an intermediate luminosity galaxy (see e.g. Sandage \& Tamman 1987).

PGC 213894, discovered to be associated to the NGC 3933 group, is an intermediate luminosity Sbc spiral with a faint disk. We estimated a total $B$-magnitude $m_{B}=15.81 \pm 1.03$ quite different from the value given by HYPERLEDA. The large error on the $B$-magnitude is attributable to the difficulty of the fit created by the residuals of the foreground $M$ star, even after its subtraction. No obvious signatures of morphological distortion are visible in the disk of the galaxy.

The high-resolution OIG images permit us also to better describe the morphology of SDSSJ115204.21+165217.3. This faint galaxy has an irregular appearance with bright knots irregularly distributed within a faint envelope. Because of this clumpy structure we did not attempted to fit any luminosity profile; to establish its total magnitudes, we preferred to fit the growing curve of the magnitude within a fixed radius $(m \leq r)$, extrapolated to infinity. Indeed, we report the total magnitudes only in Table 3. The comparison with the stellar position in GSC3 catalogues shows that knots are likely part of the galaxy, because these are extended sources.

The edge-on spiral UGC 6835 shows a warped disk structure. This feature and the NGC 3934 system of shells are the only morphological unambiguous signatures of interaction in the NGC 3933 group of galaxies.

\subsection{UV morphology and integrated properties}

In Fig. 4 we plot the NUV and FUV emission over the unsharp masking of the $R$-band OIG image. We notice that the FUV emission is centered on the nuclear part of the galaxy and the dust-lane strongly absorbs the emission in this area. The NUV emission is still in the nuclear part of the galaxy but more homogeneously distributed and extended. Furthermore, we notice some NUV knots in the northwest side of the galaxy along the edge of the shell. These NUV knots along the NW ripple of NGC 3934 offer a similarity with another shell galaxy, NGC 1210 (Marino et al. 2009). In this latter galaxy several knots, visible both in the NUV and the FUV bands, follow a tail-like structure in coincidence with a HI tail/ring. This point further supports our interpretation of these features in NGC 3934 


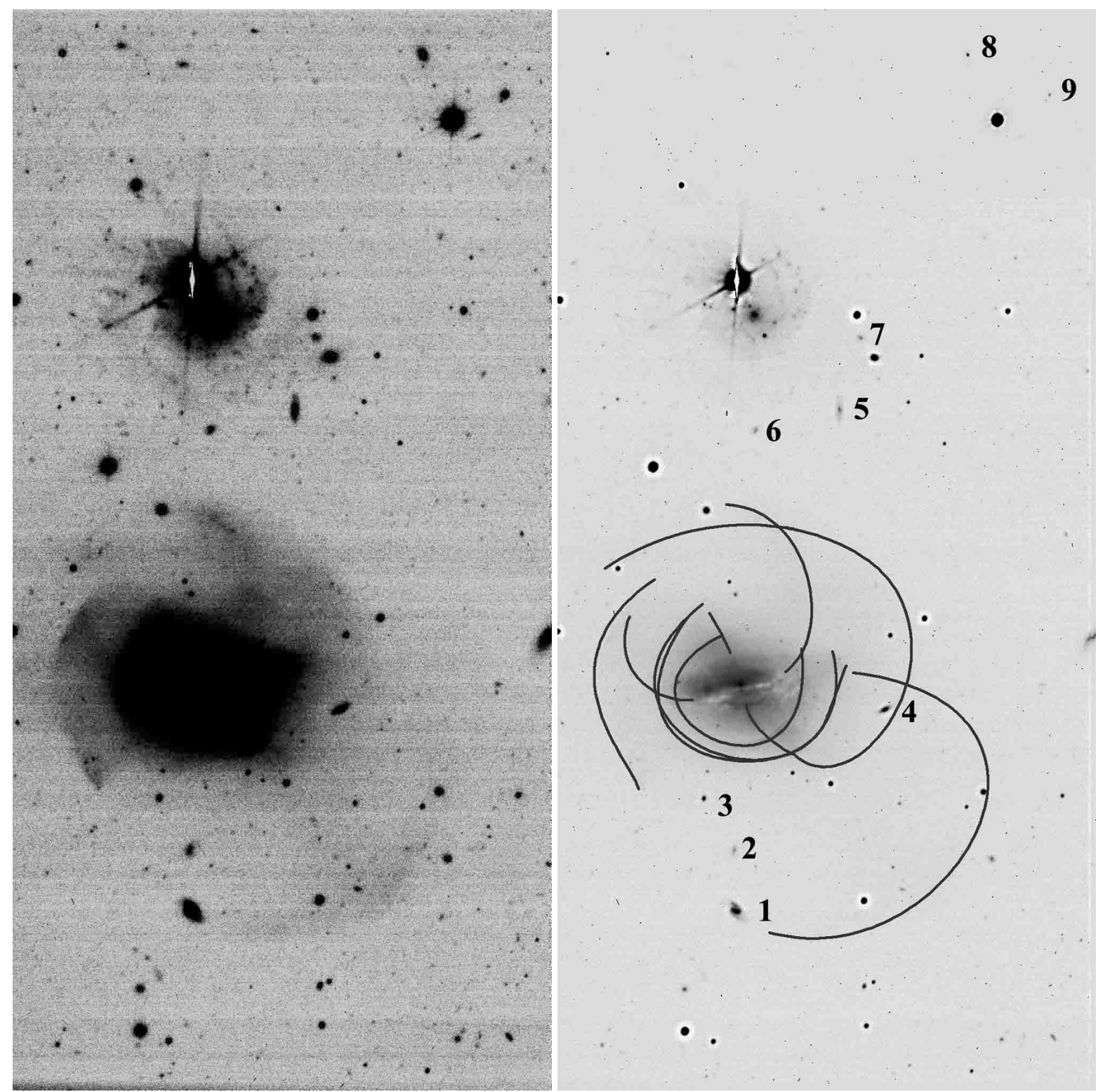

Fig. 3. Left panel: deep TNG+OIG R-band image of NGC 3934 (North is up, East to the left). Right panel: unsharp masking of the $R$ band image. Solid lines sketch the edges of the chaotic system of shells and spurs that are visible in the deep image; numbers refers to the faint galaxies listed in Table 4.

as the debris of a recent accretion event that is responsible for the shell structure.

In Table 2 we present the values of the integrated photometry in the FUV and NUV bands of GALEX. In the second column we also report the morphological classification we adopted for the galaxies in the group. In general, the morphology is not provided in the current literature (see Table 1) or is incorrect, as for NGC 3934, which we reclassified as a peculiar S0 on the basis of the present surface photometry.

In Fig. 7 (top panel) we plot the (FUV - NUV) color as a function of the morphological type. The average relationship found by Gil de Paz et al. (2007) for late-type galaxies (from spirals to irregulars) is over-plotted as a solid line. For comparison we also plot the members of Local Group Analogs (LGA) analyzed by Marino et al. (2010). These groups are mostly composed of late-type members, although two early-type are present. The galaxies in the present sample are distributed in this plane with a dispersion consistent with the findings of Gil de Paz et al. (2007) and with colors similar to groups studied by Marino et al. (2010). The (FUV-NUV) color of NGC 3934, greatly absorbed by strong dust-lanes, locates the galaxy in the region nearby early-type galaxies in LGA group.

In Fig. 7 (bottom panel) we show the NUV-S DS $S-r$ color vs. the absolute $M r$ magnitude. For comparison we also plot the set of early-type galaxies in Marino et al. (2011a), for which both GALEX and SDSS observations were available. These latter are located on the red sequence derived by Yi et al. (2005) $\left[(-0.23 \pm) 0.30 \times M_{r}+0.75\right]$ for early-type galaxies in the local universe $(0<z<0.05)$. Late-type galaxies, in particular NGC 3933 and UGC 6835, are located in the blue sequence but also in the green-valley (Salim et al. 2007). The shell galaxy NGC 3934 with its large absorption bands is also located in the green valley. Note that galaxies showing morphological signatures of interaction are located in the transitional region, the green valley, where evolving systems are found (Marino et al. 2011b).

In Fig. 8 we show the SDSS composite image of the NGC 3933 group with the isocontours of the $1.4 \mathrm{GHz}$ 


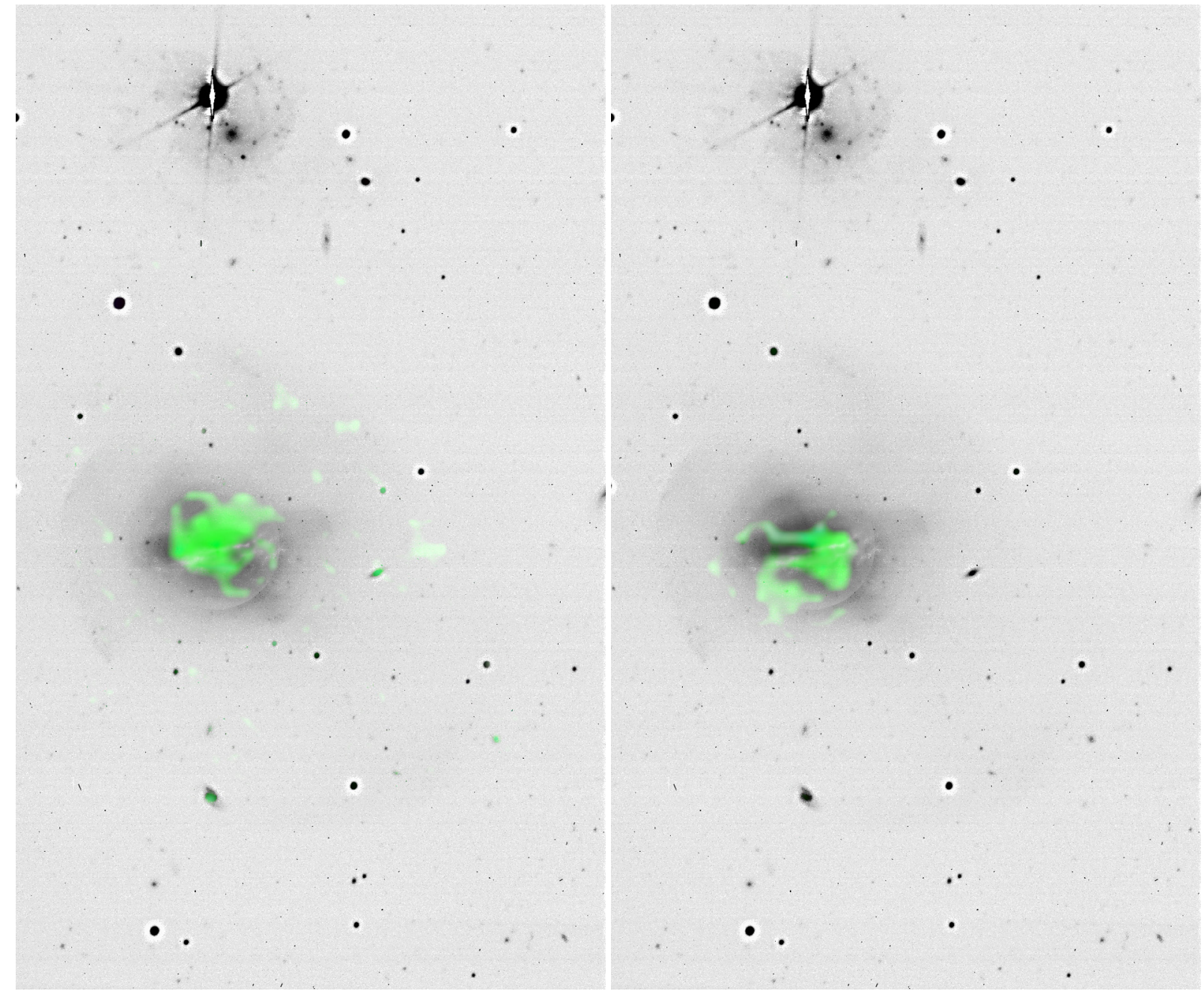

Fig. 4. Unsharp masking of the $R$-band TNG+OIG image of NGC 3934, superposed the smoothed NUV (left panel) and FUV (right panel) emission from GALEX (see text). North is at the top, East to the left.

continuum emission from the NRAO VLA Sky Survey (NVSS, Condon et al. 1998) superimposed. Two galaxies in the field are associated with significant radio emission. From Condon et al. (1998) we derive for NGC $3934 F_{1.4}=2.8 \times 10^{-28} \mathrm{~W} \mathrm{~m}^{-2} \mathrm{~Hz}^{-1}$ corresponding with our adopted distance (see Table 5) to a luminosity $L_{1.4}=8.34 \times 10^{21} \mathrm{~W} \mathrm{~Hz}^{-1}$ and for NGC $3933 F_{1.4}=3.0 \times$ $10^{-29} \mathrm{~W} \mathrm{~m}^{-2} \mathrm{~Hz}^{-1}$, corresponding to a luminosity $L_{1.4}=8.94 \times$ $10^{20} \mathrm{~W} \mathrm{~Hz}^{-1}$. The ratio $L_{1.4}(\mathrm{NGC} 3934) / L_{1.4}(\mathrm{NGC} 3933) \sim 10$ is the same as the one we found for the $60 \mu \mathrm{m}$ flux ratio. The $1.4 \mathrm{GHz}$ luminosity is insensitive to dust obscuration and for this reason is a good tracer of the star formation rate $\left(S F R_{1.4}\right)$. We adopt the calibration of Hopkins et al. (2003) and we found an $S F R=4.6 M_{\odot} \mathrm{yr}^{-1}$ for NGC 3934 and $S F R=0.83 M_{\odot} \mathrm{yr}^{-1}$ for NGC 3933. These SFR will be discussed below.

\section{Discussion}

\subsection{Group membership, kinematic and dynamic analysis of the NGC 3933 group}

The projected position and the luminosity properties of five out six galaxies, (Table 5) fulfill the criteria devised by Hickson (1982) for a compact group of galaxies. We indicate these galaxies as the "core" of the NGC 3933 group, as shown in Fig. 9, where we plot the projected distribution of galaxies within $2 \mathrm{Mpc}$ and $\pm 500 \mathrm{~km} \mathrm{~s}^{-1}$ from the average recession velocity of the group itself. The galaxies included in the larger box in Fig. 9 are PGC 139672, PGC 036501, PGC 213877, PGC 036524, NGC 3853, PGC 036561, PGC 036681, PGC 1496906, PGC 036768, UGC 06794, PGC 1538830, SDSSJ115348.42+162636.0, PGC 2806925, SDSSJ115744.10+171329.1, NGC 4014, and SDSSJ115857.18+160404.0. According to the HYPERLEDA classification, these galaxies are mainly spirals, like in the NGC 3933 group core. Ramella et al. (1994) suggest that compact configurations are continuously forming within loose groups.

Our multi-band photometric data allow us to perform a multi-band kinematical and dynamical analysis of the group. Table 5 summarizes our results computed according for the recipes given by Firth et al. (2006). All mass-related quantities are obtained by luminosity-weigthing the contribution from each member. Each galaxy is weighted by its relative luminosity evaluated from FUV to $\mathrm{r}$ magnitude converted to relative luminosity (with the following absolute solar magnitudes: $M_{\mathrm{FUV} \odot}=16.02$, $\left.M_{\mathrm{NUV} \odot}=10.15, M_{B \odot}=5.45, M_{r \odot}=4.67^{3}\right)$. The average center of mass, weighted on luminosity, shifts by about $1.5^{\prime}$ from the optical to UV bands, moving southwest from NGC 3933 towards PGC 37112. The right panel of Fig. 9 shows the galaxies

${ }^{3}$ http://mips.as.arizona.edu/ cnaw/sun.html 


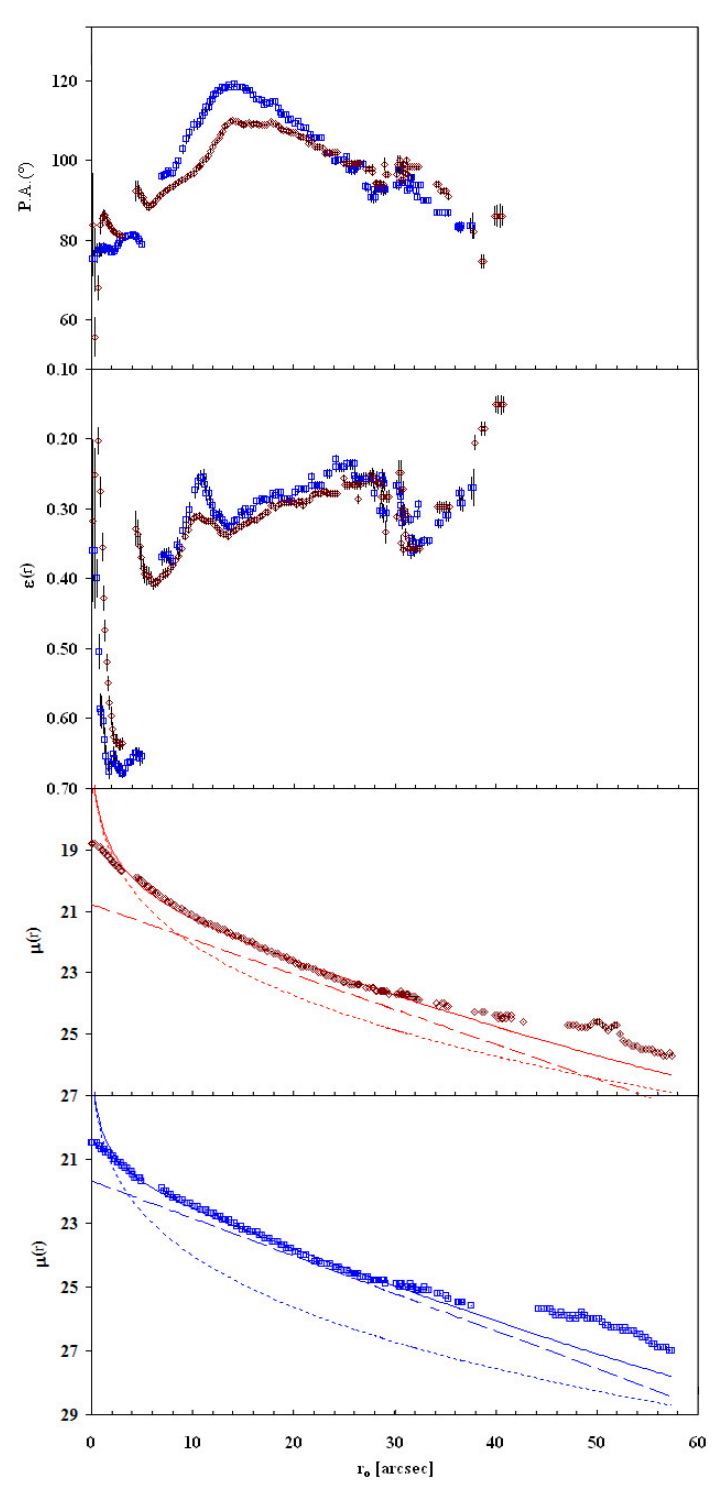

Fig. 5. NGC 3934: position angle (top panel), ellipticity (mid panel), and surface brightness (bottom panels) profiles as function of the galacto-centric distance in the $B$ - (blue open squares) and $R$ - (red open diamonds) bands obtained from the TNG+OIG observations. In addition to the global luminosity profile, the profiles of disk (dashed line) and bulge (dotted line) are also shown.

included in the harmonic radii of NGC 3933, weighted at NUV and $r$-band magnitudes.

The projected mass increases from $2.5 \pm 0.9 \times 10^{12} M_{\odot}$ in the optical bands to $7.7 \pm 2.5 \times 10^{12} M_{\odot}$ in the FUV. A crossing time of 0.04 Hubble times suggests that, at least in the central region, the group is virialized. The virial mass of the group is small, lower than $2.3 \pm 0.9 \times 10^{12} M_{\odot}$. Using this mass value, the relationship by Mathews et al. (2006) predicts a diffuse X-ray luminosity of about $10^{39} \mathrm{erg} \mathrm{s}^{-1}$ compatible with upper limits of the Rosat All Sky Survey (RASS, Voges et al. 1999). The overall group velocity dispersion on the order of $100-150 \mathrm{~km} \mathrm{~s}^{-1}$ is relatively low, but not unusual for compact configurations (see e.g. Mamon 2000). This low velocity dispersion could either argue against the virialization of the group (chance alignment with a loose group) or suggest that the tidal friction is slowing down the galaxies and bring the group toward the full coalescence.
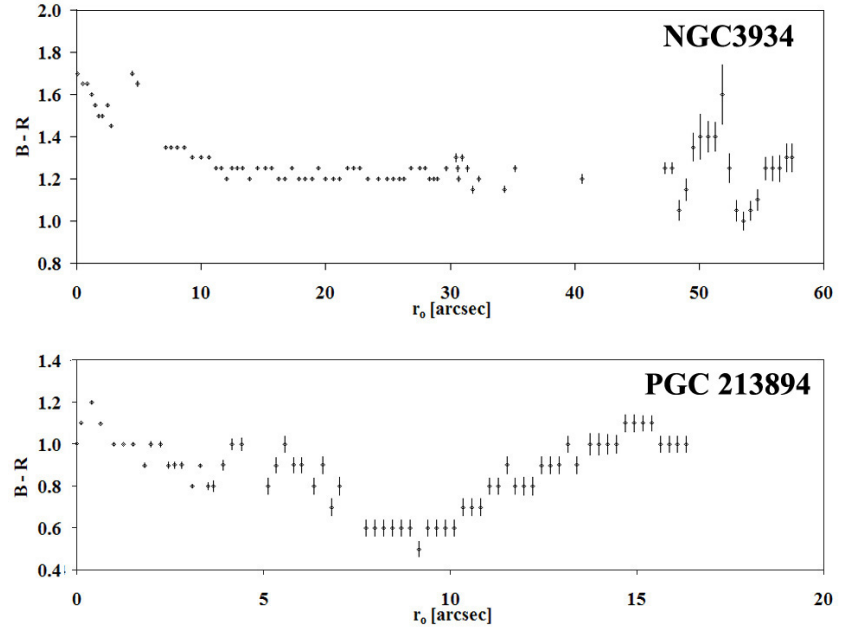

Fig. 6. Top panel: color profile of NGC 3934; bottom panel: color profile of PGC 213894.

\subsection{Comparing the data with models}

For the two brightest galaxies of this group (NGC 3933 and NGC 3934) we have extensive sets of data from the Far-UV to the FIR, they are considered as a close pair in the FIR (Geller et al. 2006) and are rich in cold gas (Galletta et al. 1997). All these data put strong constraints on the evolution of these galaxies; the dynamical analysis of the group is another important constraint for our models. With the aim to match the overall SEDs and the global properties of these galaxies in a consistent way with the dynamical properties of the group, we select two cases from a large set of SPH simulations which give us insights into the evolution of these systems and of the group itself.

Our SPH simulations of galaxy formation and evolution are starting from the same initial conditions as described in Mazzei \& Curir (2003, MC03 in the following) and Mazzei (2003) i.e., a collapsing triaxial systems initially composed of dark matter (DM) and gas in different proportions and different total masses. With respect to $\mathrm{MC} 03$, the particle resolution is enhanced here to $4 \times 10^{4}$ instead of $2 \times 10^{4}$, so there are $2 \times 10^{4}$ particle of gas and $2 \times 10^{4}$ of DM at the beginning in each new simulation.

Moreover, a set of simulations of galaxy encounters involving systems with 1:1 mass ratios and the same initial conditions as in MC03 was also performed. By seeking to exploit a wide range of orbital parameters, we carried out different simulations for each couple of interacting systems, varying the orbital initial conditions to acquire for the ideal Keplerian orbit of two equal point masses of mass equal to $10^{12}$, or $10^{13} M_{\odot}$, the first peri-center separation, $p$, equal to the initial length of the major axis of the dark matter triaxial halo, i.e. $88 \mathrm{kpc}$ for $10^{12} M_{\odot}$, or equal to $1 / 10,1 / 7$, and $1 / 5$ of the same axis for $10^{13} M_{\odot}$ encounters. For each of these separations, we changed the eccentricity to obtain hyperbolic orbits of different energy. The spins of the systems are generally parallel to each other and perpendicular to the orbital plane, so we studied direct encounters however, some cases with misaligned spins were also analyzed to deepen the effects of the system initial rotation on the results. Moreover, for a given set of encounters with the same orbital parameters we also examined the role of increasing initial gas fractions. These simulations will be fully discussed in a different paper (Mazzei 2011, in prep.).

All simulations include self-gravity of gas, stars and DM, radiative cooling, hydrodynamical pressure, shock heating, 
Table 5. Dynamical analysis of the NGC 3933 group.

\begin{tabular}{|c|c|c|c|c|c|c|c|c|c|}
\hline Band & $\begin{array}{c}\text { Center } \\
\text { of mass } \\
\text { RA Dec }\end{array}$ & $\begin{array}{c}V_{\text {group }} \\
{\left[\mathrm{km} \mathrm{s}^{-1}\right]}\end{array}$ & $\begin{array}{l}\text { Group } \\
\text { vel. disp. } \\
{\left[\mathrm{km} \mathrm{s}^{-1}\right]}\end{array}$ & $\begin{array}{l}\text { Dist. }^{+} \\
{[\mathrm{Mpc}]}\end{array}$ & $\begin{array}{l}\text { Harmonic } \\
\text { radius } \\
\text { [Mpc] }\end{array}$ & $\begin{array}{c}\text { Virial } \\
\text { mass } \\
{\left[10^{13} M_{\odot}\right]}\end{array}$ & $\begin{array}{c}\text { Projected } \\
\text { mass } \\
{\left[10^{13} M_{\odot}\right]}\end{array}$ & $\begin{array}{c}\text { Crossing } \\
\text { time } \\
\text { time } \times H_{0}\end{array}$ & $\begin{array}{c}\text { Group } \\
\text { luminosity* } \\
{\left[10^{11} L_{\odot}\right]}\end{array}$ \\
\hline$F U V$ & 177.9964216 .802330 & $3746 \pm 67$ & $165 \pm 47$ & 49.94 & $0.077 \pm 0.004$ & $0.23 \pm 0.09$ & $0.77 \pm 0.25$ & $0.04 \pm 0.02$ & $242 \pm 24.0$ \\
\hline$N U V$ & 177.9993816 .804332 & $3748 \pm 64$ & $157 \pm 44$ & 49.97 & $0.071 \pm 0.005$ & $0.19 \pm 0.09$ & $0.70 \pm($ & $4 \pm 0.02$ & $1.46 \pm 0.25$ \\
\hline$B$ & 178.0148616 .820691 & $3745 \pm 39$ & $96 \pm 27$ & 49.93 & $0.055 \pm 0.008$ & $0.06 \pm 0.03$ & $0.25 \pm 0.09$ & $0.03 \pm 0.03$ & $0.14 \pm 0.02$ \\
\hline$r$ & $178.01821 \quad 16.824264$ & $3739 \pm 41$ & $100 \pm 28$ & 49.85 & $0.053 \pm 0.005$ & $0.06 \pm 0.03$ & $0.25 \pm 0.09$ & $0.03 \pm 0.03$ & $0.19 \pm 0.02$ \\
\hline
\end{tabular}

Notes. ${ }^{+}$Distance is derived from redshift adopting $H_{0}=75 \mathrm{~km} \mathrm{~s}^{-1} \mathrm{Mpc}^{-1} ;^{*}$ group luminosities refer to their respective band.
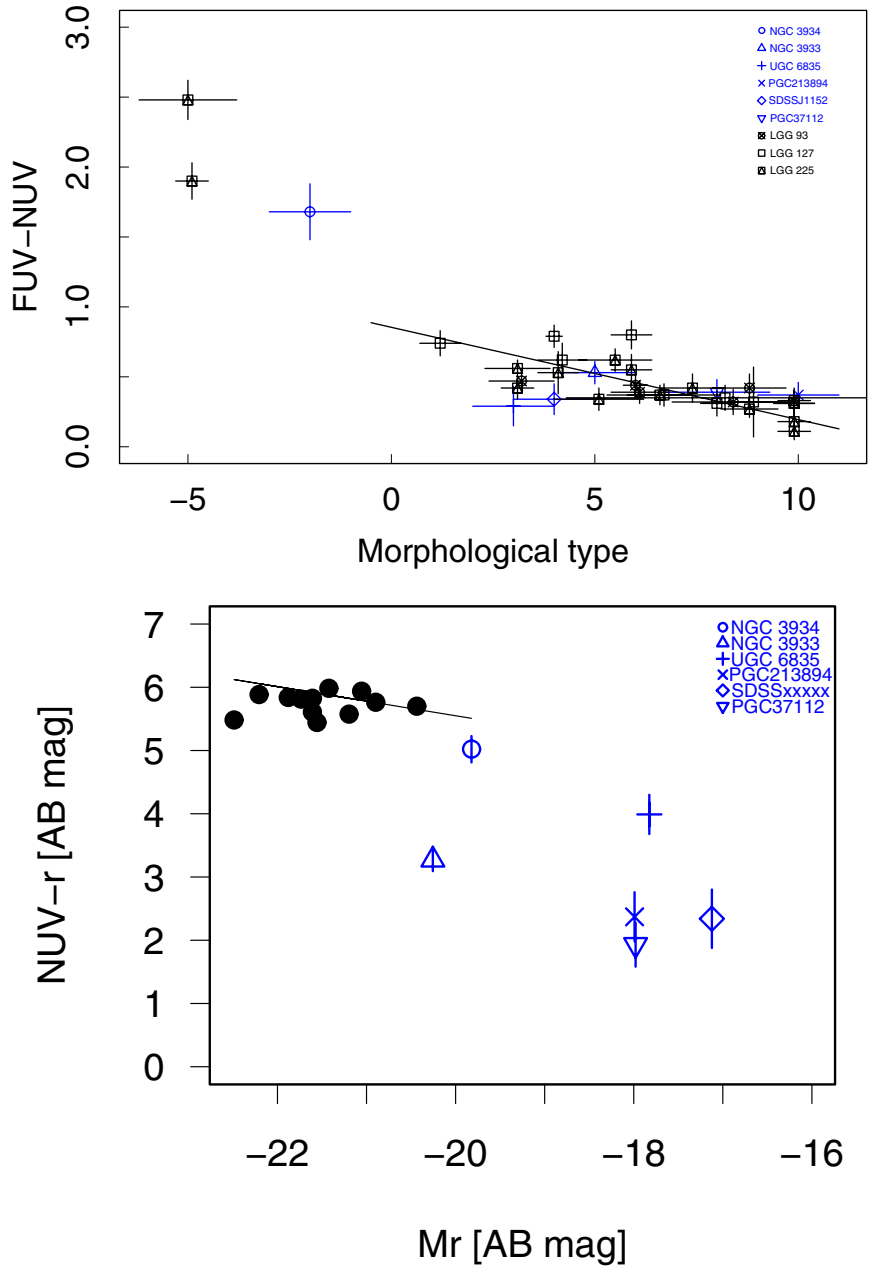

Fig. 7. Top: (FUV - NUV) color as a function of the morphological type for the six group members of the NGC 3933 group. The solid line represents the best linear fit for types $T=-0.5$ or later (i.e. spiral galaxies and irregular) in Gil de Paz et al. (2007). Members of LGA groups from Marino et al. (2010) are plotted for comparison (see text). Bottom: NUV-r vs. $M r$ color-magnitude plot for galaxies in the NGC 3933 group. The Yi et al. (2005) red sequence is shown as a solid line. Earlytype galaxies in Marino et al. (2011a) are indicated as black dots (see text).

artificial viscosity, star formation (SF) and feedback from evolved stars and type-II SNe, as in MC03.

The initial mass function (IMF) is of Salpeter type with upper mass limit $100 M_{\odot}$ and lower mass limit $0.01 M_{\odot}$ (Salpeter 1955, see MC03 and references therein for a discussion). Indeed all our simulations provide the synthetic SED at each evolutionary step. The SED accounted for chemical evolution, stellar emission, internal extinction and re-emission by dust in a self-consistent way, as described in Spavone et al. (2009), and references therein; this extends over four orders of magnitude in wavelength, i.e., from 0.1 to $1000 \mu \mathrm{m}$. So, each simulation self-consistently provides morphological, dynamic and chemophotometric evolution.

Two simulations, able to match the global properties of NGC 3934 and NGC 3933, are chosen and discussed in detail below. These point toward an independent evolution of NGC 3933 whereas NGC 3934, is the result of a major merger, whose final phase began 3 Gyr ago.

\subsubsection{NGC 3933}

We derive an adequate match of dynamical and photometric properties of NGC 3933 with a simulation of total mass $10 \times$ $10^{11} M_{\odot}$. The starting point is a triaxial collapsing system initially composed of $4 \times 10^{4}$ particles, $2 \times 10^{4}$ of gas and $2 \times 10^{4}$ of DM with a relative mass ratio 0.1 . The system is built up as described in MC03, i.e., with a spin parameter, $\lambda$, given by $|\boldsymbol{J} \| E|^{0.5} /\left(G M^{0.5}\right)$, where $E$ is the total energy, $\boldsymbol{J}$ the total angular momentum and $G$ the gravitational constant, equal to 0.06 and aligned with the shorter principal axis of the DM halo; the triviality ratio of the DM halo, $\tau=\left(a^{2}-b^{2}\right) /\left(a^{2}-c^{2}\right)$ (Warren et al. 1992), where $a>b>c$, is 0.84 with an average radius of about $500 \mathrm{kpc}$.

Figure 10 (bottom panel) compares the predicted SED with the available data, accounting for inclination between line of sight and polar axis, of 74.4 degrees (HYPERLEDA; Mazzei \& De Zotti 1992; Bekki \& Shioya 2001).

Dynamical predictions, in particular the maximum gas rotation velocity of $130 \mathrm{~km} \mathrm{~s}^{-1}$, agree with kinematic features observed for NGC 3933 (136.8 $\pm 15.2 \mathrm{~km} \mathrm{~s}^{-1}$, HYPERLEDA). The predicted average age of stellar populations is $4.5 \mathrm{Gyr}$ and the total star formation rate (SFR), $2 M_{\odot} \mathrm{yr}^{-1}$, is consistent with the value derived from the $L_{1.4 \mathrm{GHz}}$ (Sect. 3.2). This SFR is higher than expected on average for its morphological type, S0a (Kennicutt 1998), it is more similar to the average total SFR of the Milky Way (Yin et al. 2009). The $M / L_{R}$ ratio of stars is about $3 M_{\odot} / L_{\odot}$ at the optical radius (i.e., $R_{25} \simeq 9 \mathrm{kpc}$ using data from $N E D^{4}$ ) and the stellar mass $1.2 \times 10^{10} M_{\odot}$, about half of the total mass at the same radius. Figure 11 shows the $R$-band simulated map of this snapshot. The mass of gas with temperature lower than $10^{4} \mathrm{~K}$, which represents the upper limit of the cold gas mass in the system (its cooling time scale is much shorter than the snapshot time range, $0.154 \mathrm{Gyr}$ ), is $3.5 \times 10^{9} M_{\odot}$, which agrees well with the results of Galletta et al. (1997). The bolometric luminosity of the whole galaxy is $3.21 \times 10^{10} L_{\odot}$. The total mass inside $55 \mathrm{kpc}$ is about $3 \times 10^{11} M_{\odot}$, rising to $3.6 \times 10^{11} M_{\odot}$ within $77 \mathrm{kpc}$.

\footnotetext{
${ }^{4}$ http://nedwww.ipac. caltech.edu/
} 


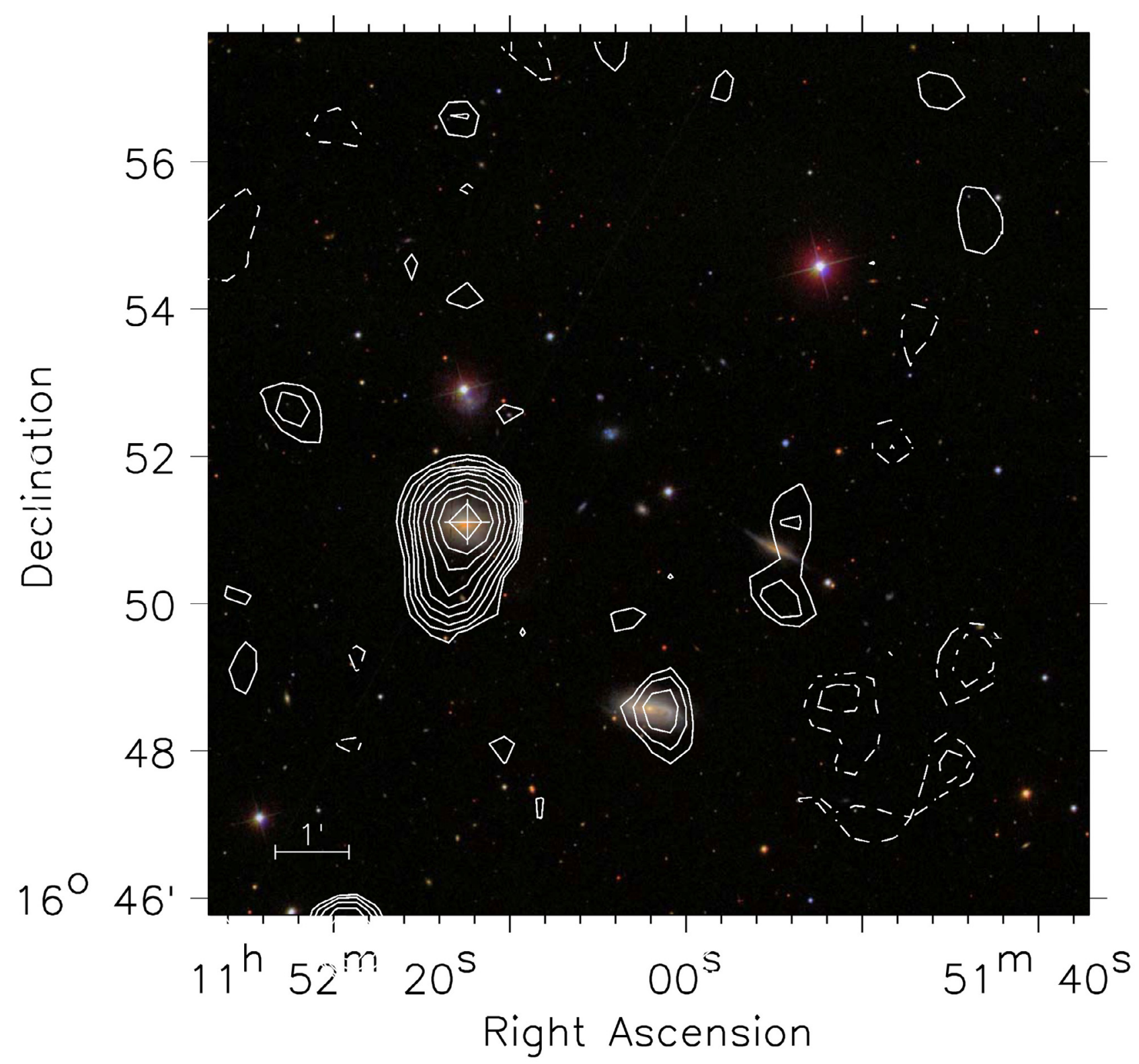

Fig. 8. Composite image from the SDSS survey of the field of the NGC 3933 group with the NVSS map superimposed. (Levels = 1, 1.4, 2, 2.8, $4 \ldots \mathrm{mJy} / b)$

The far-IR (FIR) SED is composed of a warm and a cold dust component and includes PAH molecules (Mazzei \& De Zotti 1992). Warm dust is located in regions of the high-radiation field, i.e., in the neighborhood of OB clusters, whereas cold dust is heated by the general interstellar radiation field. The energy ratio between the warm and the cold dust components in Fig. 10 (bottom panel) is 0.3 , the intensity of diffuse radiation field is about three times higher than the value used to match the Galactic FIR emission (Mazzei \& De Zotti 1992) and the warm dust temperature is about $67 \mathrm{~K}$.

\subsubsection{NGC 3934}

The whole SED and the global properties of NGC 3934 are matched well by a merger simulation with initial systems of equal total mass, $10^{13} M_{\odot}$, and a gas fraction of 0.01 ; the first peri-center separation is $175 \mathrm{kpc}$, corresponding to $1 / 10$ of the major axis, and the eccentricity 1.2. Initial star systems born in the inner regions of their halos after 2.5 Gyr from the beginning grow, changing their shapes step by step as their trajectories are approaching. Stellar systems merge in a unique configuration after 9 Gyr. However, the merger event does not complete until their centers of mass lose all their residual energy to coalesce in the center of mass of the whole system. In the meantime a shell system arises from their oscillations which on average expands and rarefies gradually, showing also re-feeding phases owing to the complex motions, shocks, viscosity and friction of several system components involved in the process of merging. The top panel of Fig. 10 compares the predicted SED with the available data accounting for 45 degrees of inclination between line of sight and polar axis (HYPERLEDA). The SED corresponds to the same age as NGC 3933, i.e. to 12.3 Gyr. However, the average age of stellar populations is younger than in the section above, 3-4 Gyr, with a higher average total SFR, about $5 M_{\odot} \mathrm{yr}^{-1}$, which remarkably agrees with the SFR we derived from the continuum radio emission at $1.4 \mathrm{GHz}$. Gas and stars draw complex structures: ripples arise if one looks at the system 


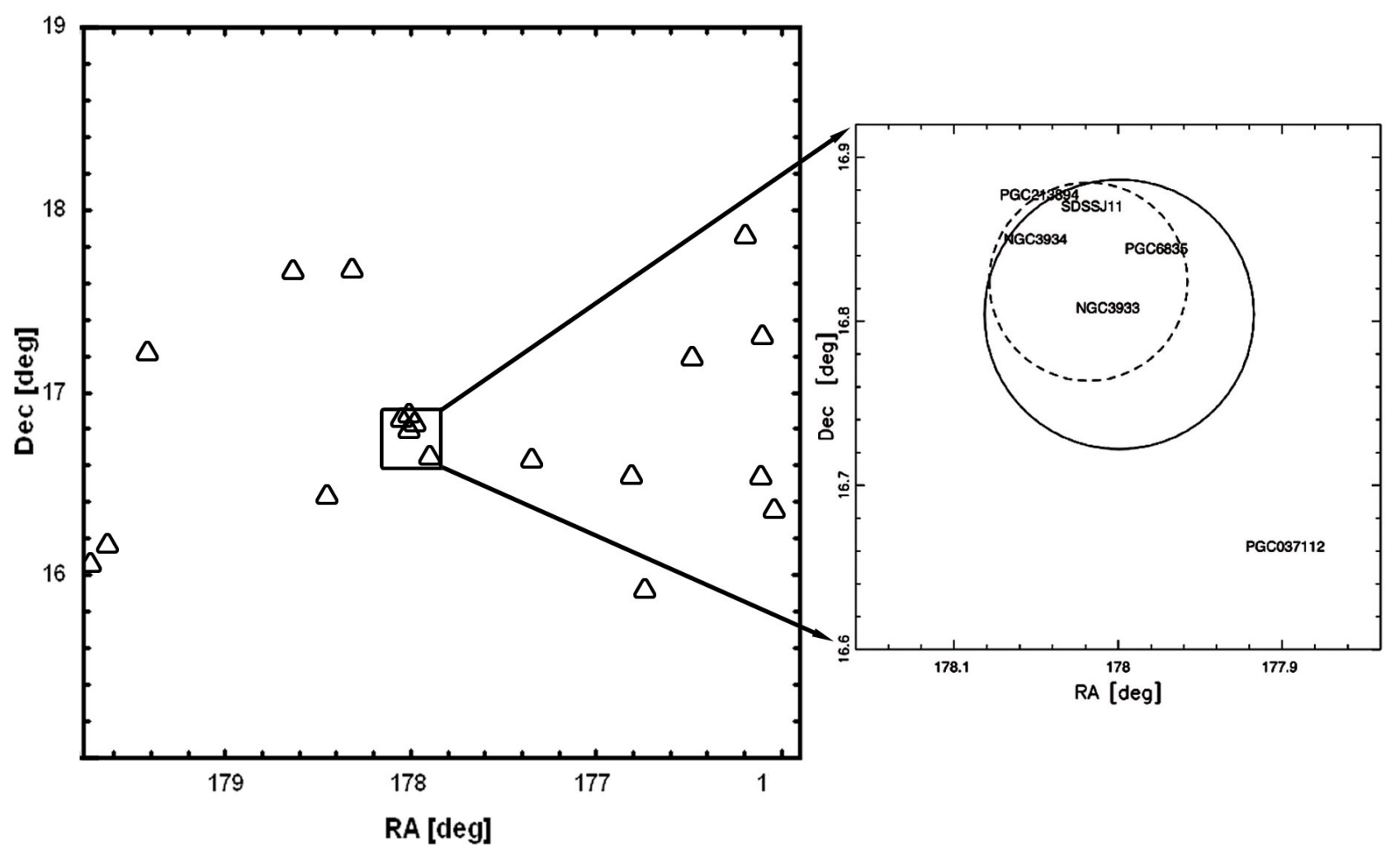

Fig. 9. Left panel: distribution of the galaxies within $2 \mathrm{Mpc}$ and the recession velocity range $3200 \leq V_{\text {hel }} \leq 4200 \mathrm{~km} \mathrm{~s}^{-1}$. Right panel: zoom on the NGC 3933 group $\left(F o V 19^{\prime} \times 19^{\prime}\right)$. The solid and dashed lines show the harmonic radius in the NUV and $r$-bands, respectively (see Table 5).

morphology in different projections. The FIR SED requires an average temperature of warm dust equal to $45 \mathrm{~K}$, a cold dust radiation field with intensity about half than that used to match the FIR SED of NGC 3933, but a higher warm-to-cold energy ratio, of 0.5 .

Figure 12 compares on the same scale a deep $R$-band image of NGC 3934 with isodensity contours of our simulation. Contours of star clusters younger than 0.05 Gyr are overplotted. They match the region of UV emission well, i.e. both the main body of the galaxy and the shell regions, as observed in Fig. 4 (left panel).

The bolometric luminosity of the whole galaxy is about twice that of NGC 3933. The total mass inside $55 \mathrm{kpc}$ is about $7 \times 10^{11} M_{\odot}$ and $11 \times 10^{11} M_{\odot}$ inside $77 \mathrm{kpc}$ which agrees well with the mass of $13 \times 10^{11} M_{\odot}$ obtained from our measured velocity dispersion (see Sect. 2.1) using the relation $M=$ $5 \times \sigma^{2} R_{\mathrm{e}} / G$ Bertin et al. (2002).

The dynamical results reported in Table 4 are compatible with the estimates of masses and radii provided by the above simulations of NGC 3933 and NGC 3934.

\section{Summary and conclusions}

We analyzed the multi-wavelength properties of NGC 3934 and its environment. The multi-band photometric analysis permits us to study in detail the morphology and the SED of NGC 3934, the second brighter member of the group, and to investigate the nature of this galaxy which is supposed to be a polar-ring galaxy (Whitmore et al. 1990).
Our study shows that NGC 3934 is not a polar-ring galaxy, as suggested by the literature. The peculiar morphology of this galaxy is caused by a prominent dust-lane structure and by a wide type-II shell structure. The shell system together with the presence of the strong dust-lane suggest that NGC 3934 is the product of a "wet" merging episode. This is confirmed also by the presence of some NUV knots along the NW ripple of NGC 3934 similar to those detected by GALEX in another shell galaxy, NGC 1210. These features are the debris of a recent accretion event that is responsible for the shell structure (Marino et al. 2009).

Our spectroscopic study of PGC 213894 allows us to add this galaxy to the bright members of the group whose projected core is composed of five galaxies, with Hickson's compact group characteristics.

Our multi-band photometric data allow us to perform a dynamical analysis of the group (Table 4). The relatively short crossing times indicate that the group is probably virialized. The low velocity dispersion could either argue against the virialization of the group (chance alignment with a loose group) or suggest that the tidal friction is slowing down the galaxies and which brings the group toward the full coalescence. The virial masses of the group are small, lower than $2.3 \pm 0.9 \times 10^{12} M_{\odot}$.

We performed and analyzed a large set of SPH simulations to match the morphology and the global properties of the brighter optical members of the group, namely NGC 3933 and NGC 3934, and to give insights into the group evolution. The simulation matching NGC 3933, a typical spiral in the group, is consistent with an unperturbed evolution of this system with an SFR similar to that of the Milky Way. On the other hand 

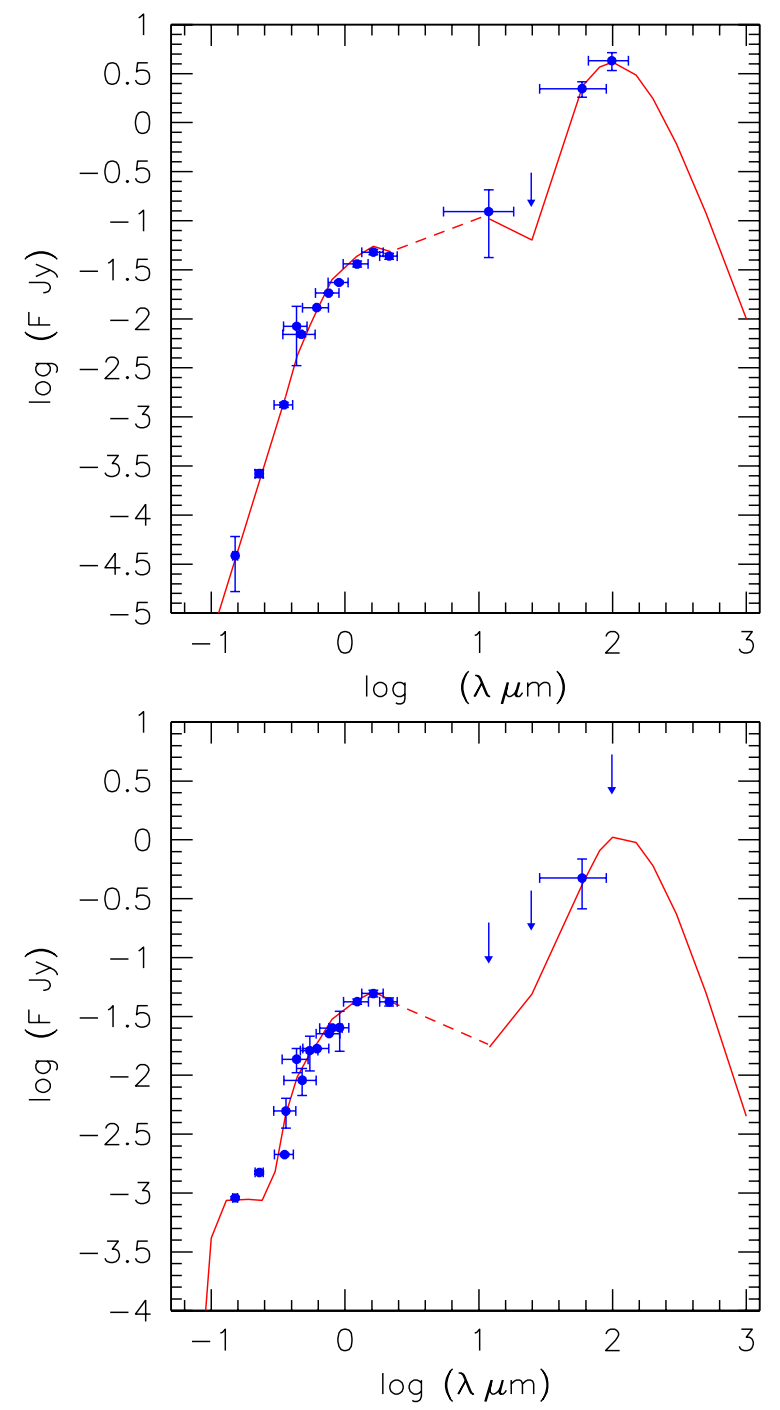

Fig. 10. Top: continuous line (red) shows the prediction of our model (see text) for NGC 3934, (blue) filled circles correspond to data from Table 2 for FUV and NUV, from Table 3 for the $B$ - and $R$ - and from NED for the remaining bands. Arrows show upper limits; error bars account for band width and $3 \sigma$ uncertainties. Bottom: symbols are the same as in the top panel, but for NGC 3933.
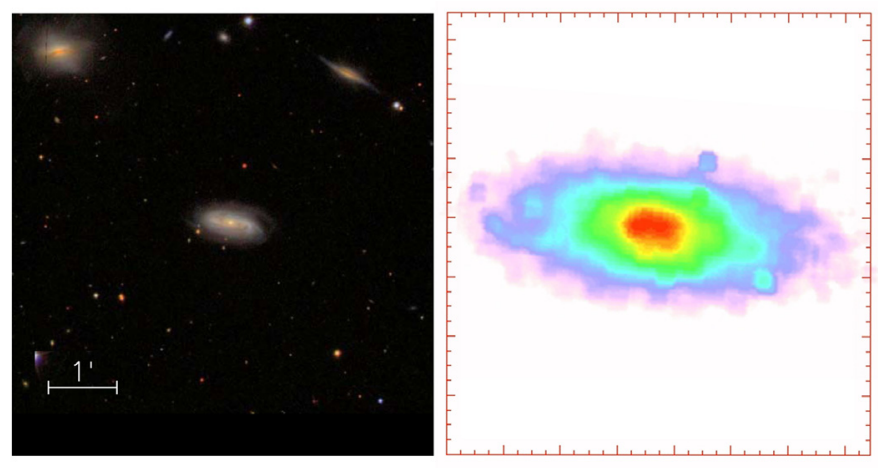

$410^{-5}$ $1.210^{-3}$

Fig. 11. Color-composite SDSS image of NGC 3933 group (left) centered on the spiral galaxy NGC 3933 is compared on the same scale with the $R$-band map from our simulation ( $r i g h t$ ); the map includes forty equi-spaced levels and a density contrast of three hundred normalized to the total flux in the map. Note that the observed galaxy (left panel) corresponds to the inner (red) region in the right panel.

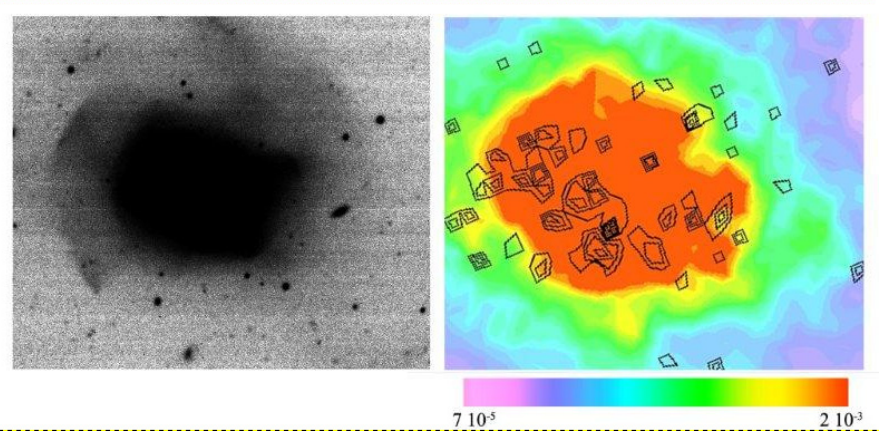

Fig. 12. Deep $R$-band image of NGC 3934 (left) compared on the same scale with isodensity contours (right) from our simulation (see text). The simulated image includes twenty-six no-equispaced levels to emphasize the shells: seven equispaced levels from $7 \times 10^{-5}$ to $1 \times 10^{-4}$, eighteen equispaced levels from $1.5 \times 10^{-4}$ to $1 \times 10^{-3}$ and then the last level, $2 \times 10^{-3}$ normalized to the total counts in the map. Black contours in the right panel correspond to star clusters 0.05 Gyr old or younger.

NGC 3934 appears to be the result of a major merger (see also Weil \& Hernquist 1993), whose final phase began 3 Gyr ago. Our simulation points at a "wet" merger, i.e. a merger where dissipation plays a meaningful role on the evolution, as observations suggest. Moreover, the simulation performed is able to account for both the whole SED of this galaxy from the UV to FIR spectral range, and for the morphology, including the shell structure and the distribution of the UV emission.

Since their discovery (Malin \& Carter 1983), shell galaxies are noticed to inhabit low-density environments, specially avoiding clusters. One remarkable exception is NGC 4552 in Virgo. NGC 3934 could represent an example of a shell galaxy in a dense (at least in projection) galaxy association. The presence of NGC 3934 makes this group an evolving galaxy association, probably at an early stage of its evolution, as suggested by the nearly unperturbed morphologically and photometric nature of its spiral members.

Acknowledgements. We tank the anonymous referee for useful comments. We acknowledge the financial contribution from the agreement ASI-INAF I/009/10/0. GALEX is a NASA Small Explorer, launched in April 2003. GALEX is operated for NASA by California Institute of Technology under NASA contract NAS-98034. This work is based on GALEX data from GI program 59 and archival data. The data were obtained from MAST (http:// galex.stsci.edu). This research has made use of the SAOImage DS9, developed by Smithsonian Astrophysical Observatory and of the NASA/IPAC Extragalactic Database (NED) which is operated by the Jet Propulsion Laboratory, California Institute of Technology, under contract with the National Aeronautics and Space Administration. We acknowledge the usage of the HYPERLEDA database (http://leda.univ-lyon1.fr). Funding for the SDSS and SDSSII has been provided by the Alfred P. Sloan Foundation, the Participating Institutions, the National Science Foundation, the US Department of Energy, the National Aeronautics and Space Administration, the Japanese Monbukagakusho, the Max Planck Society, and the Higher Education Funding Council for England. The SDSS Web Site is http://www.sdss.org/. The SDSS is managed by the Astrophysical Research Consortium for the Participating Institutions. The Participating Institutions are the American Museum of Natural History, Astrophysical Institute Potsdam, University of Basel, University of Cambridge, Case Western Reserve University, University of Chicago, Drexel University, Fermilab, the Institute for Advanced Study, the Japan Participation Group, Johns Hopkins University, the Joint Institute for Nuclear Astrophysics, the Kavli Institute for Particle Astrophysics and Cosmology, the Korean Scientist Group, the Chinese Academy of Sciences (LAMOST), Los Alamos National Laboratory, the Max-Planck-Institute for Astronomy (MPIA), the Max-Planck-Institute for Astrophysics (MPA), New Mexico State University, Ohio State University, University of Pittsburgh, University of Portsmouth, Princeton University, the United States Naval Observatory, and the University of Washington. We tanks the anonymous referee for useful comments. 


\section{References}

Bertola, F., Bettoni, D., Rusconi, L., \& Sedmak, G. 1984, AJ, 89, 356 Bertola, F., Galletta, G., \& Zeilinger, W. W. 1985, ApJ, 292, L51

Bekki, K. 1997, ApJ, 490, L37

Bekki, K. 1998, ApJ, 499, 635

Bekki, K. 2009, MNRAS, 399, 2221

Bekki, K., \& Shioya, Y. 2001, ApJS, 134, 241

Bianchi, L. 2009, Ap\&SS, 320, 11

Bournaud, F., \& Combes, F. 2003, A\&A, 401, 817

Bertin, G., Ciotti, L., \& Del Principe, M. 2002, A\&A, 386, 149

Condon, J. J., Cotton, W. D., Greisen, E. W., et al. 1998, AJ, 115, 1693

Coziol, R., Iovino, A., \& de Carvalho, R. R. 2000, AJ, 120, 47

de Vaucouleurs, G. 1948, Ann. Astrophys., 11, 247

de Vaucouleurs, G., de Vaucouleurs, A., Corwin, H. G., et al. 1991, RC3

Dupraz, C., \& Combes, F. 1986, A\&AS, 166, 53

Dressler, A. 1980, ApJ, 236, 351

Firth, P., Evstigneeva, E. A., Jones, J. B., et al. 2006, MNRAS, 372, 1856

Freeman, K. C. 1970, ApJ, 160, 811

Galletta, G., Sage, L. J., \& Sparke, L. S. 1997, MNRAS, 284, 773

Geller, M. J., Kenyon, S. J., Barton, E. J., Jarrett, T. H., \& Kewley, L. J. 2006, AJ, 132, 2243

Gil de Paz, A., Boissier, S., Madore, B. F., Seibert, M., \& Joe, Y. H. 2007, ApJS, 173,185

Gunn, J. E., \& Gott, J. R. III 1972, ApJ, 176, 1

Hickson, P. 1982, ApJ, 255, 382

Hopkins, A. M., Miller, C. J., Nichol, A. J., et al. 2003, ApJ, 599, 971

Jedrzejewski, R. I. 1987, MNRAS, 226, 747

Kennicutt, R C., Jr. 1998, ARA\&A, 36, 189

Landolt, A. U. 1983, AJ, 88, 439

Malin, D., \& Carter, D. F. 1983, ApJ, 274, 534

Mamon, G. A. 2000, in Small Galaxy Groups, ASP Conf. Ser., 209, 217

Marino, A., Iodice, E., Tantalo, R., et al. 2009, A\&A, 508, 1235

Marino, A., Bianchi, L., Rampazzo, R., Buson, L. M., \& Bettoni, D. 2010, A\&A, 511, A29
Marino, A., Rampazzo, R., Bianchi, L., et al. 2011a, MNRAS, 411, 311

Marino, A., Bianchi, L., Rampazzo, R., et al. 2011b, Ap\&SS, 13

Martin, D. C., Fanson, J., Schiminovich, D., et al. 2005, ApJ, 619, L1

Mathews, W., \& Brighenti, F. 2006, ApJ, 652, L1

Mazzei, P. 2003, MmSAI., 74, 498

Mazzei, P., \& Curir, A. 2003, ApJ, 591, 784 (MC03)

Mazzei, P., \& De Zotti, G. 1992, A\&A, 256, 45

Moore, B., Lake, G., \& Katz, N. 1998, ApJ, 495, 139

Morrissey P., Schiminovich, D., Barlow, T. A., et al. 2005, ApJ, 619, L7

Oemler, A. Jr. 1974, ApJ, 194, 1

Prieur, J.-L. 1990, International Conference on Dynamics and Interactions of Galaxies, 72

Ramella, M., Diaferio, A., Geller, M. J., \& Huchra, J. P. 1994, AJ, 107, 1623

Rampazzo, R., Alexander, P., Carignan, C., et al. 2006, MNRAS, 368, 851

Reshetnikov, V., \& Sotnikova, N. 1997, A\&A, 325, 933

Salim, S., Rich, R. M., Charlot, S., et al. 2007, ApJS, 173, 267

Salpeter, E. E. 1955, ApJ, 121, 161

Sandage, A. R., \& Tammann, G. 1987, A Revised Shapley Ames Catalogue of Bright Galaxies, Carnegie, Washington (RSA)

Sargent, W. L. W., Schechter, P. L., Boksenberg, A., \& Shortridge, K. 1977, ApJ, 212,326

Schweizer, F., Whitmore, B. C., \& Rubin, V. C. 1983, AJ, 88, 909

Smith, P. S., Jannuzi, B. T., \& Elston, R. 1991, ApJS, 77, 67

Spavone, M., Iodice, E., Calvi, R., et al. 2009, MNRAS, 393, 317

van Driel, W., Combes, F., Arnaboldi, M., \& Sparke, L. S. 2002, A\&A, 386, 140

Varnas, S. R., Bertola, F., Galletta, G., Freeman, K. C., \& Carter, D. 1987, ApJ, 313, 69

Voges, W., Aschenbach, B., Boller, Th., et al. 1999, A\&A, 349, 389

Weil, M., \& Hernquist, L. 1993, ApJ, 405, 142

Warren, M., Quinn, P., Salmon, J., \& Zurek, W. 1992, ApJ, 399, 405

Whitmore, B. C., Lucas, R. A., McElroy, D. B., et al. 1990, AJ, 100, 1489 (PRC)

Yi, S. K., Yoon, S.-J., Kaviraj, S., et al. 2005, ApJ, 619, L111

Yin, J., Hou, J., Prantzos, N., et al. 2009, A\&A, 505, 497

Zepf, S. E. 1993, ApJ, 407, 448 